\title{
When a CAR becomes a tank
}

T cells can specifically target tumour cells through expression of chimeric antigen receptors (CARs), which are synthetic receptors consisting mostly of an extracellular antigen recognition domain and intracellular T cell activation and co-stimulation domains. CAR $\mathrm{T}$ cells have proved very effective in the treatment of patients with some haematological malignancies; however, treatment of solid tumours has remained challenging, partly owing to the immunosuppressive tumour microenvironment. In turn, inhibition of the immunosuppressive tumour microenvironment with checkpoint blockade therapy has shown remarkable responses in patients with a range of solid tumours, albeit with some severe side effects. Rafiq et al. have now combined both approaches in a single type of 'armoured' CAR T cell that secretes immune checkpoint blockade fragments and could provide improved efficacy and safety over dual-therapy regimens.

The authors had already described armoured CAR T cells, whereby CAR T cells had been modified to express immuno-modulatory ligands or to secrete cytokines. On the basis of these findings, the authors decided to use this platform

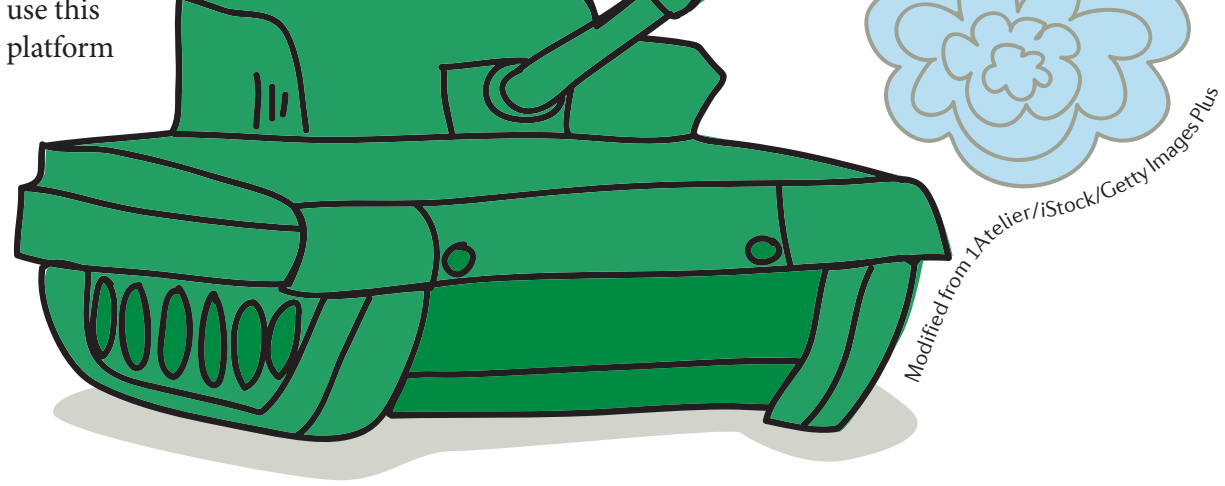

to create CAR T cells that could secrete single-chain variable fragments (scFvs) of monoclonal antibodies (mAbs) that block immune checkpoint signalling. First, the authors generated second-generation CAR constructs containing domains that recognize the antigens CD19 (found on B cell malignancies) or MUC16 (expressed on ovarian and pancreatic cancer cells) fused to mouse CD28 and CD3 $\zeta$ T cell signalling domains. They then modified this construct to include an $\mathrm{scFv}$ from an anti-mouse programmed cell death 1 (PD1) mAb and transduced primary mouse $\mathrm{T}$ cells with this construct.

To test the efficacy of PD1-blocking scFv-secreting CAR T cells (anti-PD1-armoured CAR T cells), the authors treated C57BL/6 mice injected with ovarian carcinoma ID8 cells. Treatment enhanced survival compared with treatment with the MUC16 CAR T cell controls. However, this survival benefit was comparable to that observed upon treatment

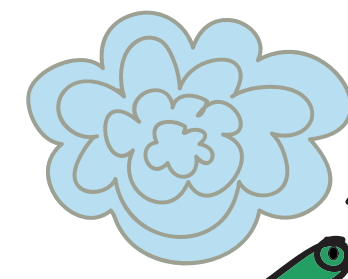

with MUC16 CAR T cells in combination with anti-mouse PD1 mAbs. Long-term surviving mice treated with the anti-PD1-armoured CAR T cells had detectable CAR T cells in the bone marrow more than 120 days post-tumour inoculation, and these mice were able to mount an antitumour response when re-challenged with ID8 tumour cells compared with untreated mice.

The authors then modified human CAR T cells encoding a CD19-targeted or MUC16-targeted CAR to secrete an anti-human PD1 scFv, called E27. In mouse models of human B cell cancers or peritoneal carcinomatosis, mice treated with E27-secreting CD19 CAR T cells or MUC16 CAR T cells, respectively, showed enhanced survival compared with mice treated with the corresponding CAR T cells alone. Moreover, mice treated with E27-secreting MUC16 CAR T cells showed enhanced survival benefit compared with mice treated with a combination of MUC16 CAR T cells and an anti-human PD1 mAb. Importantly, further experiments showed that, unlike checkpoint blockade mAbs, E27 secreted by CAR T cells remained localized in the tumour microenvironment and was not present systemically, which may result in less immune-related adverse events.

These results open new possibilities to make $\mathrm{T}$ cell therapies more effective, particularly against solid tumours, by using scFvs that target other checkpoint molecules, such as LAG3 or CTLA4.

M. Teresa Villanueva

ORIGINAL ARTICLE Rafiq, S. et al. Targeted delivery of a PD-1-blocking scFv by CAR-T cells enhances anti-tumor efficacy in vivo. Nat. Biotechnol. 36, 847-856 (2018) 